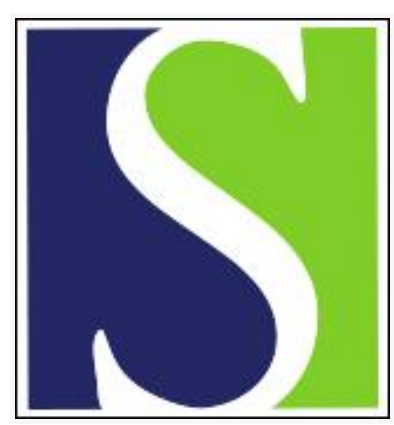

Scand J Work Environ Health 1999;25(2):144-150

https://doi.org/10.5271/sjweh.417

Issue date: Apr 1999

Work stress and menstrual patterns among American and Italian nurses

by Hatch MC, Figa-Talamanca I, Salerno S

The following article refers to this text: 2015;41(2):194-203

Key terms: menstrual cycle; nurse; reproductive health; stress

This article in PubMed: www.ncbi.nlm.nih.gov/pubmed/10360470

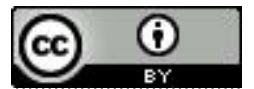




\title{
Work stress and menstrual patterns among American and Italian nurses
}

\author{
by Maureen C Hatch, PhD, ${ }^{1}$ Irene Figa-Talamanca DrPH, ${ }^{2}$ Silvana Salerno, MD
}

\begin{abstract}
Hatch MC, Figa-Talamanca I, Salerno S. Work stress and menstrual patterns among American and Italian nurses. Scand J Work Environ Health 1999;25(2):144-150.

Objectives This study assessed whether job stress alters menstrual patterns among nurses working in 2 different settings: a tertiary care hospital in New York ( 99 nurses) and a university hospital in Rome ( 25 nurses).

Methods Data on menstrual patterns were collected by a daily diary in which the nurses recorded their basal body temperature (BBT) and their menstrual bleeding status for a 3-month period. The BBT curves were used to classify cycles as biphasic or monophasic, and as adequate or inadequate with respect to the luteal phase. Job stress was evaluated by both objective (environmental and work characteristics) and subjective (perceived stress) criteria.

Results The American nurses, especially those assigned to high stress units, had an increased risk for long and monophasic cycles [relative risk (RR) $4.3,95 \%$ confidence interval $(95 \% \mathrm{CI}) 1.1-16.2$ and RR $5.5,95 \% \mathrm{CI} 1.2-$ 25.5, respectively]. Among those who perceived their stress at work to be high or reported strenuous work activity, the risk for longer cycles was also raised (RR $2.3,95 \% \mathrm{CI} 0.6-8.0$ and $\mathrm{RR} 1.6,95 \% \mathrm{CI} 0.7-4.2$, respectively). Luteal phase inadequacy followed the same pattern. Similar trends were observed in the Italian data. In addition, the rotating shiftwork pattern prevalent in the Italian group was possibly associated with higher rates of short cycles and inadequate luteal phases when compared with those of nurses working fixed shifts either day or night.

Conclusions Menstrual function may be affected by stressful work conditions.
\end{abstract}

Key terms menstrual cycle, nurses, reproductive health, stress.

Various types of environmental stress may interfere with endocrine function and produce menstrual cycle disorders $(1,2)$. Clinicians have long described stress-related menstrual problems with a variety of endocrine profiles, especially a lowering of gonadotropin and estrogen secretion suggestive of hypothalamic involvement (3). Given the proximity of the neural cortex and the hypothalamus, interactions between hormones on the central nervous system and reproductive hormones should not be unexpected. While such interactions have occurred in situations of extreme stress, whether disturbances in menstrual pattern can be caused by situations of everyday life is still open to question. A recent study of nurses in Sweden (4) observed an association between perceived job strain and an elevation in plasma prolactin. Menstrual function was not assessed, but, typically, as prolactin levels progressively increase, so does luteal phase inadequacy, anovulation, and, ultimately, amenorrhea (5). Shift work, which alters the natural circadian rhythms governing hormone secretion and the synchronization of ovulatory events, has been found to modify menstrual profiles (6).

Only a few studies have examined the effects of work conditions on menstrual function. In a large survey of American nurses (7), job stress was associated with a 3fold increase in amenorrhea and other menstrual abnormalities. Perimenstrual symptoms (especially lower abdominal pain) have been found among nurses in Cana$\mathrm{da}$, specifically associated with the lifting of weights (8). Two other studies - of female industrial workers in Canada (9) and China (10) - observed an association between unfavorable work conditions, self-reported job stress, and menstrual dysfunction. In Europe, 2 recent studies found reduced fecundability among women on shift work $(11,12)$ but no clear effect on menstrual function.

Empirical data on occupational stress and the menstrual cycle are still limited, in part because measurement

1 Division of Epidemiology, Mount Sinai School of Medicine, New York, New York, United States.

2 University La Sapienza, Rome, Italy

3 ENEA, Rome, Italy.

Reprint requests to: Dr Maureen C Hatch, Division of Epidemiology, Department of Community and Preventive Medicine, Mount Sinai School of Medicine, One Gustave L Levy Place, Box 1043, New York, NY 10029-6574, USA. [E-mail: maureen.hatch@mount_sinai.org] 
difficulties, subjective factors, and cultural attitudes have hampered the assessment of both putative work stressors and the type and severity of menstrual dysfunction. In order to address these problems, we undertook a longitudinal study in which both stress and menstrual abnormalities were evaluated by more detailed and objective methods than those used in previous occupational studies.

The study population consisted of nurses - a group with exposure to a variety of stressful work conditions, including strenuous physical activity, psychological stress, and irregular and inconvenient work schedules. In the United States, the National Institute for Occupational Safety and Health has identified nurses as a group whose levels of job stress are elevated to above occupational norms $(13,14)$. Apart from exposure, nurses' training and experience help to assure adherence to research protocols, in this case involving daily diaries and basal body temperature charts. The goal of the study was to determine whether the levels of stress the women experienced on the job would lead to measurable alterations in menstrual function.

\section{Subjects and methods}

\section{Subjects}

The principal study site was a tertiary care hospital in New York City, chosen because of the high role demands on nurses. With the prospect of extending the range of stressful work conditions and sociocultural perceptions of stress, the protocol was also applied to a small sample of nurses from the University Hospital in Rome, Italy. Information was obtained from 99 nurses recruited in New York City, and 30 nurses recruited in Rome. In the latter group, the final number of subjects who adhered to the protocol was 25 , and it was impossible to increase the number of participants because of limited resources. Virtually all the subjects were Caucasian. Restriction to one ethnic group seemed advisable in light of evidence suggesting ethnic differences in the circulating levels of steroid hormones (5).

We targeted recruitment at particular units that were judged from record-based data at each hospital to represent relatively high or low stress environments. Once the target units were identified, introductory letters were left in the office mailboxes of all the nurses who were employed there and under 50 years of age. The letter invited them to participate in a screening questionnaire to determine eligibility. The study was presented to the nurses as a study of life-style factors, hormonal patterns, and menstrual function. Nurse managers, instructors, and nurse researchers were approached along with registered staff nurses; licensed practical nurses were not enrolled.
Eligible women were those still menstruating, not using or planning to use oral contraceptives or intrauterine devices, with no diagnosis of diabetes, polycystic ovarian disease, endometriosis, thyroid disease, adrenal or pituitary hormone disorder, and with no current anemia or clotting problems. Sixty percent of those screened in New York and 54\% in Rome proved to be eligible. Because we could not be sure whether the nurses actually received our introductory letters, it is difficult to calculate accurate response rates. To be conservative, we assumed that all the distributed letters reached their targets and have estimated participation according1y. Thus, among the eligible women, the participation rates were $61.3 \%$ in New York and $62 \%$ in Rome. The time and inconvenience of the daily protocol were the usual reasons given for refusal.

Overall, the New York nurses were older than the Italian nurses ( 34 years and 32 years, respectively) (table 1). They were less likely to be current smokers ( $16 \%$ versus $50 \%$ ) and more likely to drink alcohol (81\% versus $27 \%$ ); the levels of smoking and drinking in the study population were moderate, however.

\section{Study instruments}

A base-line interview was administered to all the participants to obtain data on social, demographic, and reproductive characteristics, work history, current work conditions and exposures, and general health status. Beginning on day 1 of menses following the intake interview, menstrual function was monitored by 2 tools: (i) basal body temperature (BBT) and (ii) menstrual records. The daily diary, where BBT and menstrual bleeding status were recorded, consisted of a core set of 19 questions, including a question about the perceived level of work stress. Every 7 th day the diary also included additional questions (eg, level of job activity). Still more items were added to form a cycle diary, completed on day 1 of the

Table 1. Selected sociodemographic characteristics of the New York and Roman nurses.

\begin{tabular}{lcc}
\hline Characteristic & $\begin{array}{c}\text { New York } \\
(\mathrm{N}=99)\end{array}$ & $\begin{array}{c}\text { Rome } \\
(\mathrm{N}=25)\end{array}$ \\
\hline Mean age (years) & $34^{\mathrm{a}}$ & $32^{\mathrm{b}}$ \\
Married (\%) & 40 & 36 \\
Parity (\%) & & \\
None & 59 & 68 \\
One & 12 & 8 \\
Two or more & 29 & 24 \\
Life-style factors (\%) & & \\
Current smokers & 16 & 50 \\
Never smokers & 61 & 40 \\
Ex-smokers & 23 & 10 \\
Coffee drinkers (some coffee) & 75 & 90 \\
Alcohol drinkers (some alcohol) & 81 & 27 \\
\hline
\end{tabular}

Standard deviation 6.6 .

- Standard deviation 8.1. 
next menstrual cycle. Altogether 8835 diaries were compiled by the 99 New York nurses (corresponding to 297 completed menstrual cycles), and 1924 daily diaries were compiled by the 25 Roman nurses (corresponding to 72 completed cycles).

\section{Definition of variables}

Menstrual function. The intraindividual cycle lengths varied little and were therefore averaged over the $3 \mathrm{cy}$ cles studied. Women were classified as having short cycles when the mean duration of the cycles was equal to or shorter than 25 days and as having long cycles when the mean cycle length was equal to or longer than 33 days.

The ovulatory pattern of each subject was evaluated using the basal body temperature (BBT) technique described by McCarthy \& Rockette in 1983 (15). The technique involves smoothing the BBT curve by replacing the temperature at the $i$ th point with the average of the 3 temperatures at points $\mathrm{I}, \mathrm{I}-1, \mathrm{I}+1$. A running average tends to eliminate date-to-day variability and presents a more stable point from which to measure the thermal shift. We considered a graph to be biphasic if the smoothed curve showed a shift in BBT from a lower to a higher phase by transecting the average cycle temperature for at least 7 days. Biphasic graphs were presumed to be ovulatory. If no temperature shift could be seen, the BBT graph was classified as monophasic and the cycle was considered anovulatory. For the purposes of this study women with all biphasic cycles were classified as having a normal ovulatory pattern, while those with only monophasic cycles were considered to have an anovulatory pattern;

Table 2. Selected work characteristics and attitudes toward work reported by the New York and Roman nurses.

\begin{tabular}{lcc}
\hline Characteristic & $\begin{array}{c}\text { New York } \\
(\mathrm{N}=99)\end{array}$ & $\begin{array}{c}\text { Rome } \\
(\mathrm{N}=25)\end{array}$ \\
\hline Type of shift (\%) & & \\
8 hours & 29 & 100 \\
$9-10$ hours & 21 & \\
12 hours & 51 & \\
Day shift only & 81 & \\
Night shift only & 19 & 100 \\
Rotating shift & - & 10 \\
Overtime work (mean weekly hours) & 3 & \\
Level of job activity & & 72 \\
Reporting strenuous & & \\
physical activity & & \\
$>50 \%$ of the time (\%) & 53 & \\
Perceived stress & & 38 \\
High (\%) & & 36 \\
Job satisfaction (\%) & 46 & 32 \\
High & & \\
Medium & 35 & \\
Low & 56 & \\
\hline
\end{tabular}

a Stress levels were based on the responses to a 1 - to 5-point stress scale recorded in the daily dairy for a 3-month period. Women whose overall mean score was $>3.0$ were classified as "high" stress. those with some biphasic and some monophasic cycles were classified as having an irregular ovulatory pattern.

The adequacy of the luteal phase was defined by the elapsed time between the occurrence of ovulation and the first day of the next menstrual cycle, with ovulation identified as the day the BBT graph registered the thermal shift. Cycles where the luteal phase lasted for at least 7 days were defined as "adequate", and cycles of shorter duration were considered "inadequate". Women having some adequate and some inadequate luteal phase cycles were classified as "irregular" in respect to the luteal phase.

Job stress. Work-related stress was examined using 4 indicators. The primary measure presented in this report is environmental work stress, based on objective ratings of the immediate work environment developed using the following 3 dimensions: (i) patient care demands, such as average acuity (deaths, orders not to resuscitate), number of boarders and difficult cases, psychiatric consults and prevalence of emergency admissions; (ii) system issues, including overtime work required, frequency of unplanned changes of schedule, staff vacancies and new policies and procedures; (iii) milieu indicators, such as staff rotation and turnover and staff relationships. Judgments about stressful environments were made a priori, by a panel of nurse managers and nurse clinicians; these judgments were primarily based on archival materials. Although workplace conditions such as violence or threats of violence (16) may affect nurses in other settings, these conditions were not present at our study sites. Examples of units rated high on stress were neurosurgery and pediatric oncology. Since the measure of environmental work stress omits the effect of individual appraisal, we also analyzed a measure of perceived stress derived from the subjects' self-reports. Perceived job stress was defined as high if the woman's score on a 5-point scale was above the mean for all the subjects. Physical job stress was assessed by the subjects' responses to a question about the amount of work time spent in strenuous activity; more than $50 \%$ was deemed high physical stress.

All of the Italian nurses worked a rotating shift, while all of the New York nurses worked a fixed shift. The Roman nurses worked the equivalent of one extra shift per week in overtime (mean 9.8 hours) compared with an average of 2.7 hours of overtime in New York. Roman nurses were more likely than the New York nurses to report strenuous work activity ( $72 \%$ and $53 \%$, respectively). Both groups found work to be a greater source of stress than life outside work. The proportion of nurses with high work stress scores was close to $50 \%$ in both settings, but the nurses in Rome were 4 times as likely to report low job satisfaction ( $43 \%$ and $10 \%$, respectively) (table 2). 


\section{Data analysis}

All the data were coded, entered, and analyzed using SPSS (statistical package for the social sciences) (17). We computed the overall frequency of short and long cycles, of monophasic cycles, and of luteal phase inadequacy in the various subgroups (New York versus Rome, high versus low stress, etc), adjusting for the effect of age on cycle characteristics using the direct standardization method. Differences in the frequency of menstrual cycle characteristics were tested for statistical significance by computing the standard error of the difference of 2 proportions. Multivariate analyses of work-related stress and menstrual patterns were conducted, with the results expressed as relative risks (RR) and their 95\% confidence intervals $(95 \% \mathrm{CI})$. We evaluated the role of possible confounders other than age - in particular smoking and drinking habits - by conducting stratified analyses, but the results did not differ between the subjects reporting some smoking or drinking and those reporting no smoking or alcohol consumption.

\section{Results}

\section{Menstrual patterns}

The overall mean cycle lengths among the New York and Roman nurses were 29 and 28 days (table 3). In the New York group, the age-adjusted frequency of long cycles was approximately $10 \%$, while $20 \%$ of the subjects were estimated to have short cycles. Among the Roman nurses almost a third (29\%) qualified as having short cycles according to the study definition.

Nine percent of the New York nurses and $16 \%$ of the Roman nurses appeared to have been anovulatory in all the study cycles. The frequency of presumed luteal phase inadequacy was $6 \%$ among the New York nurses and
$12 \%$ among the Roman nurses; in both groups luteal defects were concentrated in the older age groups.

We also examined the interrelationships among cycle length, ovulatory patterns, and luteal phase adequacy (data not shown). Among the New York nurses whose BBT profile suggested irregular ovulation, most (66\%) also had abnormal cycle lengths. In the Roman group, this proportion reached $90 \%$. A similar overlap was observed for the association between luteal phase inadequacies and cycle length.

\section{Relation of work stress to menstrual patterns}

Compared with nurses working in hospital units classified as low stress environments, those in high stress units showed a 4- to 5-fold risk of long cycles and monophasic cycles (RR 4.3, 95\% CI 1.1-16.2, and RR 5.5, 95\% CI 1.2-25.5, respectively) (table 4). Among those who perceived their stress at work to be high, the risk for long cycles was also raised (RR 2.3.95\% CI 0.6-8.0) (table 4). Among nurses reporting strenuous job activity, long

Table 3. Age-adjusted prevalence of menstrual irregularities among the New York and Roman nurses.

\begin{tabular}{lcc}
\hline Type of irregularity & $\begin{array}{c}\text { New York } \\
(\mathrm{N}=99)\end{array}$ & $\begin{array}{c}\text { Rome } \\
(\mathrm{N}=25)\end{array}$ \\
\hline Cycle length & & \\
Mean (days) & $29^{\mathrm{a}}$ & $28^{\mathrm{b}}$ \\
Short cycles $(\%)$ & 20 & 29 \\
$\quad$ Long cycles (\%) & 10 & 6 \\
Ovulatory pattern & & \\
$\quad$ Monophasic (\%) & 9 & 16 \\
Irregular (\%) & 37 & 16 \\
Luteal phase & & \\
Inadequate (\%) & 6 & 12 \\
Irregular (\%) & 37 & 49 \\
\hline
\end{tabular}

a Standard deviation 2.2

- Standard deviation 2.3

" $\leq 25$ days.

d $\geq 33$ days.

Table 4. Prevalence (\%), rate ratios (RR), and $95 \%$ confidence intervals $(95 \% \mathrm{Cl})$ for menstrual irregularities among the New York nurses ( $N=99)$ exposed to high environmental work stress ${ }^{a}$ and the prevalence $(\%)$, RR, and $95 \% \mathrm{Cl}$ for menstrual irregularities in nurses reporting high perceived job stress.

\begin{tabular}{|c|c|c|c|c|c|c|c|c|}
\hline \multirow[t]{2}{*}{ Type of irregularity } & \multicolumn{4}{|c|}{ Environmental stress } & \multicolumn{4}{|c|}{ Perceived stress } \\
\hline & High & Low & $\mathrm{RR}^{\mathrm{b}}$ & $95 \% \mathrm{Cl}$ & High & Low & $\mathrm{RR}^{\mathrm{b}}$ & $95 \% \mathrm{Cl}$ \\
\hline \multicolumn{9}{|l|}{ Cycle length } \\
\hline Short (\%) & 8 & 11 & 0.7 & $0.2-27.7$ & 7 & 16 & 0.4 & $0.1-1.7$ \\
\hline Long $(\%)$ & 20 & 5 & 4.3 & $1.1-16.2$ & 18 & 8 & 2.3 & $0.6-0.8$ \\
\hline \multicolumn{9}{|l|}{ Ovulatory pattern } \\
\hline Monophasic (\%) & 26 & 5 & 5.5 & $1.2-25.5$ & 6 & 9 & 0.7 & $0.2-2.9$ \\
\hline Irregular $(\%)$ & 37 & 28 & 1.4 & $0.5-3.0$ & 34 & 36 & 0.9 & $0.4-2.2$ \\
\hline \multicolumn{9}{|l|}{ Luteal phase } \\
\hline Inadequate $(\%)$ & 8 & 8 & 1.0 & $0.2-4.3$ & 2 & 8 & 1.0 & $0.0-2.3$ \\
\hline Irregular $(\%)$ & 22 & 28 & 0.8 & $0.3-2.0$ & 34 & 43 & 0.8 & $0.4-1.8$ \\
\hline
\end{tabular}

a The work units were classified as high or low stress according to criteria described in the Methods section of the text.

- The RR values were adjusted for age. 
Table 5. Prevalence (\%), rate ratios (RR), and 95\% confidence intervals $(95 \% \mathrm{Cl})$ for menstrual irregularities among the New York nurses reporting "high" and "low" job activity levels.

\begin{tabular}{|c|c|c|c|c|}
\hline \multirow[t]{2}{*}{ Type of irregularity } & \multicolumn{2}{|c|}{ Job activity level } & \multirow[t]{2}{*}{$\mathrm{RR}^{\mathrm{a}}$} & \multirow[t]{2}{*}{$95 \% \mathrm{Cl}$} \\
\hline & $\begin{array}{c}\text { High } \\
(N=50)\end{array}$ & $\begin{array}{c}\text { Low } \\
(N=49)\end{array}$ & & \\
\hline \multicolumn{5}{|l|}{ Cycle length } \\
\hline $\begin{array}{l}\text { Short }(\%) \\
\text { Long }(\%)\end{array}$ & $\begin{array}{r}8 \\
15\end{array}$ & $\begin{array}{l}15 \\
10\end{array}$ & $\begin{array}{l}0.5 \\
16\end{array}$ & $\begin{array}{l}0.2-1.3 \\
0.7-4.2\end{array}$ \\
\hline \multicolumn{5}{|l|}{ Ovulatory pattern } \\
\hline $\begin{array}{l}\text { Monophasic (\%) } \\
\text { Irregular (\%) }\end{array}$ & $\begin{array}{r}8 \\
38\end{array}$ & $\begin{array}{l}10 \\
40\end{array}$ & $\begin{array}{l}0.8 \\
0.9\end{array}$ & $\begin{array}{l}0.3-3.6 \\
0.5-2.4\end{array}$ \\
\hline \multicolumn{5}{|l|}{ Inadequate } \\
\hline $\begin{array}{l}\text { Iuteal adequacy (\%) } \\
\text { Irregular luteal phase (\%) }\end{array}$ & $\begin{array}{r}4 \\
40\end{array}$ & $\begin{array}{r}7 \\
29\end{array}$ & $\begin{array}{l}0.5 \\
1.4\end{array}$ & $\begin{array}{l}0.3-3.2 \\
0.5-3.5\end{array}$ \\
\hline
\end{tabular}

adjusted for age.

Table 6. Prevalence (\%) of irregularities in cycle length, ovulatory patterns, and luteal adequacy by type of work shift. ${ }^{a}$

\begin{tabular}{|c|c|c|c|}
\hline \multirow[t]{2}{*}{ Type of irregularity } & \multicolumn{2}{|c|}{$\begin{array}{c}\text { New York } \\
(\mathrm{N}=99)\end{array}$} & \multirow{2}{*}{$\begin{array}{c}\begin{array}{c}\text { Rome } \\
(\mathrm{N}=25)\end{array} \\
\text { Rotating shift }\end{array}$} \\
\hline & $\begin{array}{c}\text { Day shift } \\
(\mathrm{N}=83)\end{array}$ & $\begin{array}{l}\text { Night shift } \\
\qquad(\mathrm{N}=16)\end{array}$ & \\
\hline \multicolumn{4}{|l|}{ Cycle length } \\
\hline Short & 10 & 19 & 29 \\
\hline Normal & 76 & 81 & 64 \\
\hline Long & 15 & - & 6 \\
\hline \multicolumn{4}{|l|}{ Ovulatory pattern } \\
\hline Monophasic (\%) & 10 & 5 & 16 \\
\hline Irregular (\%) & 35 & 45 & 29 \\
\hline Biphasic (\%) & 56 & 52 & 55 \\
\hline \multicolumn{4}{|c|}{ Luteal phase adequacy } \\
\hline Inadequate (\%) & 5 & 5 & 12 \\
\hline Irregular (\%) & 33 & 38 & 48 \\
\hline Adequate (\%) & 63 & 57 & 40 \\
\hline
\end{tabular}

a The New York nurses worked fixed day or night shifts. All the Rome nurses worked rotating shifts.

cycles were somewhat more common (RR 1.6, 95\% CI $0.7-4.2$ ) and luteal phase inadequacy somewhat less common (RR $0.5,95 \%$ CI $0.3-3.2$ ) than for nurses reporting less strenuous work (table 5). Finally, the small number of nurses working a rotating shift had somewhat higher rates of short cycles and inadequate luteal phases in comparison with nurses working either fixed day or fixed night shifts, but the differences were not statistically significant (table 6).

\section{Discussion}

The study was designed to assess whether job stress alters menstrual patterns in otherwise healthy female workers. The menstrual profiles of the nurses studied showed the same decline in cycle length and variability with increasing age that has been seen in samples unselected for employment $(18,19)$. In addition we found evidence that menstrual function was affected by work in a stressful environment. Among the nurses in the United States, we observed increased risks for long cycles and presumed anovulatory cycles in those assigned to high-stress work units. While the Italian data are too sparse for a formal analysis, the same pattern was present. We also found a modest association between long cycles and perceived job stress although the result was not statistically significant.

The relatively weak association between high perceived job stress and altered menstrual function may be due in part to random measurement error since we assessed perceived stress with a single question rather than a multi-item scale. As the question was included in the daily diary, some degree of habituation may have occurred, a phenomenon that typically leads to underestimation. Given the nature of the study outcomes, any errors that are present are likely to be nondifferential, biasing results towards the null. In an earlier study of work conditions among Swedish nurses (4), job strain was perceived to be higher among those working in the emergency room than among those in the child psychiatry and pediatric outpatient units; this finding suggests that perceptions of job stress tend to be realistic. In summary, in our data, work stress, measured either in objective or subjective terms, appears to lead to cycle lengthening (RR 4.3 and RR 2.3, respectively).

Preliminary observations by one of us (SS), an occupational health physician trained in the method of organizational congruences (20), indicated that the work of nurses differed in the 2 study hospitals, both qualitatively and quantitatively. Hospital work in Rome involved monotonous and repetitive tasks, lack of time for direct patient care, lack of time for staff to exchange information, high physical work load, and limits on professional growth. In New York, on the contrary, routine tasks were mostly done by auxiliary personnel, allowing nurses to dedicate themselves mostly to direct patient care (21). In addition, New York nurses were required to work much fewer hours of overtime each week. In spite of these marked differences, the perception of job stress among the New York and Roman nurses was similar. This similarity may help to explain why the measure of perceived stress shows a weaker association with menstrual dysfunction than the objective measure. Although cognitive appraisal is generally thought to be an important component of a stress response (22), such a large discrepancy between external stressors and the perception of stress raises questions about the utility of the measure in the Italian sample.

In addition to job stress overall, we evaluated 2 specific work stressors, strenuous activity and rotating shift work. A relationship between strenuous activity, longer cycles, and amenorrhea has been observed consistently 
among professional athletes and regular exercisers (23, 24). That activity on the job should also affect the length of the menstrual cycle is therefore not surprising, particularly when the job is as physically demanding as nursing. Nonetheless, a recent study of hospital employees in Denmark found only weak evidence for a relationship between tiring physical work and menstrual dysfunction, although fecundability was reduced (12). It should be noted that the observed association in our study between strenuous job activity and long cycles was independent of perceived job stress. However, this association with cycle length was modest and not statistically significant.

The trend we observed between rotating shift work and menstrual dysfunction has also been seen previously. Among women in a meat packing plant, work schedule variability was associated with irregular menstrual cycles and amenorrhea (9). The nurses on shift work in our study showed an increased risk for short cycles. Since rotating work between day and night will tend to interfere with circadian rhythms and thereby disrupt hormonal balance $(6,25)$, alterations in the menstrual cycle are not surprising. In contrast to food industry workers who experienced amenorrhea, our nurses on rotating shifts showed the same proportion of biphasic, presumably ovulatory cycles as nurses working a fixed shift; this finding would argue against decreased levels of estrogen as a cause of the changes we observed among our shift workers.

In order to interpret the data from this study, several issues need to be reviewed. First, did selection bias arise from the relatively low participation rates? Among the women eligible for the study, time and inconvenience of data collection were cited as the principal reasons for refusing to enroll; this occurrence suggests that nonparticipants perceived themselves to be busier and perhaps more stressed than those who participated. In addition, the rates of presumptive anovulatory cycles and luteal phase inadequacy among the participants appeared to be higher than expected, raising the possibility that subjects might have been self-selected for fecundability problems. This is speculative, however, since there are few normative data on ovulation frequency and luteal phase defects for women in the age group we studied. Moreover, the consistency between the findings from this and earlier studies concerning the relationship of age to cycle length provides some reassurance that the response rate has not biased the results.

In addition to selection bias, confounding must be considered. Smoking was relatively uncommon in this sample of nurses. The Roman nurses were more often current smokers, but none of them smoked more than 10 cigarettes a day. Furthermore, we found no clear association between this level of smoking and menstrual cycle end points. Thus, while smoking is a stress-related behavior that can reduce ovarian reserve (26), it did not confound the analysis of job stress in this study. Leisuretime exercise proved to be infrequent among the nurses, possibly because their level of job activity was fairly high, and those who did exercise did so only at low-tomoderate levels. Perhaps as a result, we observed no association between exercise and cycle length or variability. Body size did not relate to menstrual function either, Similarly, an earlier study of menstrual function found stress to be a more important determinant of cycle length than either weight or physical activity (27).

Some of the analyses presented have used all $3 \mathrm{cy}$ cles contributed by each study subject, an approach that can affect the estimation of variance (28). As a consequence, the confidence intervals may actually be wider than they appear. In addition, the analysis was cross-sectional in nature and therefore unable to distinguish coincidence from cause. However, reverse causality (eg, long cycles causing job stress) does not seem a likely explanation.

The internal consistency in our analyses tends to support the findings. Using several definitions of job stress, we saw an association between job stress and long cycles. In addition, there were similar trends in the Italian sample as in the larger group of American nurses, although the 2 groups were exposed to different work conditions. The higher frequency of short cycles and luteal phase abnormalities among Roman nurses is consistent with an effect of rotating shift work, to which they were all exposed and which was not a factor in the American hospital. The results seem to be biologically consistent as well, long cycles tending to be associated with anovulation and short cycles relating to a short luteal phase. In terms of external evidence, the only other study we know that considers ovarian function in nurses (7) also found an association with job stress: specifically, an association between self-reported job stress and menstrual dysfunction, defined as amenorrhea, altered cycle length and altered length of bleed. Associations between menstrual dysfunction and strenuous activity have also been reported previously (9).

Our study adds to the evidence concerning factors that can affect menstrual function among female workers. Further study will be needed to determine what health implications the observed alterations in menstrual function may have, either for conceiving or sustaining a pregnancy, or for influencing the risk of certain chronic diseases. In the case of breast cancer, recent research suggests that both short and long menstrual cycles, indicative of anovulation, are associated with a reduction in risk $(30,31)$. The data presented, derived from a sample of nurses, do appear to implicate job stress, strenuous job activity, and possibly rotating shift work in the disruption of the menstrual cycle of otherwise healthy Caucasian workers. 


\section{Acknowledgments}

We are grateful to Patricia Lewis, Marilyn Bookbinder, and Linda Kennelly for their contribution to the fieldwork among nurses in New York City and to Kristen Friedman, Rachel Levine, Shyly Amarasinghe, and Dr $\mathrm{Bu}-\mathrm{Tian} \mathrm{Ji}$ for their assistance with all aspects of the study. Dr Melissa Begg provided statistical advice. The Roman researchers wish to thank Marina Simeoni, Bianca M. Scicchitano, and Claudia Manno for helping with the data collection and analysis and all the nurses participating in the study.

This study was supported by a grant from the United States National Institute for Occupational Safety and Health (R01 OHO2885) and by the Italian National Research Council (no 94.04357).

\section{References}

1. Armstrong DT. Environmental stress and ovarian function. Biol Reprod 1986;34:29-39.

2. Berga SL. How stress can affect ovarian function. Contemporary OB/GYN 1993;87-94.

3. Warren M. The effects of altered nutritional states, stress and systemic illness on reproduction in women. In: J. Vaitukaitis, editor. Clinical reproductive endocrinology, New York (NY): Elsevier, 1982;177-206.

4. Theorell T, Ahlberg-Hulten G, Jodko M, Sigala F, de la Torre $B$. Influence of job strain and emotion on blood pressure in female hospital personnel during work hours. Scand J Work Environ Health 1993;9:313-8.

5. Speroff L, Glass RH, Kase NG. Clinical gynecologic endocrinology and infertility. Baltimore (MD): Williams and Wilkins, 1989;213-32.

6. Turek F, Swann J, Earnest D. Role of the circadian system in reproductive phenomena. Recent Prog Horm Res 1984;40:143-77.

7. Shortridge L, Lemasters G, Valanis B. Menstrual cycles in nurses handling antineoplastic drugs. Cancer Nurs 1995; 18:439-44.

8. Tissot F, Messing K. Perimenstrual symptoms and working conditions among hospital workers in Quebec. Am J Ind Med 1995;27:511-22.

9. Messing K, Saurel-Cubizolles M-J, Bourgine M, Kaminski M. Menstrual-cycle characteristics and work conditions of workers in poultry slaughterhouses and canneries. Scand J Work Environ Health 1992;18:302-9.

10. Christiani DC, Niu T, Xu X. Occupational stress and dysmenorrhea in women working in cotton textile mills. Int Occup Environ Health 1995;1(1);9-15.

11. Bisanti L, Olsen J, Basso O, Thonneau P, Karmaus W. The European Study Group on Infertility and Subfecundity: a European Multicenter Study. J Occup Environ Med 1996;38:352-8.

12. Florack EIM, Zielhuis GA, Rolland R. The influence of occupational physical activity on the menstrual cycle and fecundability. Epidemiology 1994;5:14--8.
13. McLauney MA, Hurrell JJ. Control, stress and job satisfaction in Canadian nurses. Work Stress 1988;2:217-24.

14. Sauter S, Murphy L, Hurrell J. Prevention of work-related psychological disorders: a national strategy proposed by the National Institute of Occupational Safety and Health. Am Psychol 1990;45:1146-58.

15. McCarthy JJ, Rockette HE. A comparison of methods to interpret the basal body temperature graph. Fertil Steril 1983;39(5):640-6.

16. Arnetz JE, Arnetz BB, Petterson IL. Violence in the nursing profession: occupational and lifestyle risk factors in Swedish nurses. Work Stress 1996;10:119-27.

17. Marijia J Norusis. SPSS/PCT for the IBM PC/XT/AT. Chicago (IL): SPSS Inc, 1986.

18. Chiazze L, Braye FT, Macisco JJ, Parker MP, Duffy BJ. The length and variability of the human menstrual cycle. JAMA 1968;203:377-80.

19. Treloar AE, Bounton RE, Behn BG, Brown BW. Variation of the human menstrual cycle through reproductive life. Intl $J$ Fertil 1967;12:77-126.

20. Maggi B. Lavoro organizzato e salute (Organization and wellbeing). Tirrenia Stampatori, Torino Maggio 1991:254.

21. Salerno S, Figa-Talamanca I, Hatch M. Application of the method of organizational congruences to assess work stress among hospital nurses in two different countries. In: 24th International Congress of Occupational Health, Nice, France, 1993.

22. Lazarus RS, Folkman S. Stress, appraisal and coping. New York (NY): Springer, 1984.

23. Baker ER, Mathur RS, Kirk RF, Williamson HO. Female runners and secondary amenorrhea: correlation with age, parity, mileage, and plasma hormonal and sex-hormone bindingglobulin concentrations. Fertil Steril 1981;36:183-7.

24. Glass AR, Deuster PA, Kyle SB, Yahiro JA, Vigersky RA, Schoomaker EB. Amenorrhea in Olympic marathon runners. Fertil Steril 1987;48:740-5.

25. Jewett M, Kronauer R, Czeisler C. Light-induced suppression of endogenous circadian amplitude in humans. Nature 1991;350:59-62,

26. Sharara FI, Beatse SN, Leonardi MR, Navot D, Scott RT Jr. Cigarette smoking accelerates the development of diminished ovarian reserve as evidenced by the clomiphene citrate challenge test. Fertil Steril 1994;62:257-62.

27. Harlow S, Matanowski $\mathrm{G}$. The association between weight, physical activity, and stress and variation in the length of the menstrual cycle. Am J Epidemiol 1991;133:38 - 49.

28. Zeger S, Liang K-Y. An overview of methods for the analysis of longitudinal data. Stat Med 1992;11:1825-39.

29. Cooper GS, Sandler DP, Wheland EA, Smith KR. Association of physical and behavioral characteristics with menstrual cycle patterns in women age 29-31 years. Epidemiology 1996;7:624-8.

30. Garland M, Hunter DJ, Colditz GA, Manson JE, Stampfer MJ, Spiegelman D, Sprizer F, Willett WC. Menstrual cycle characteristics and history of ovulatory infertility in relation to breast cancer risk in a large cohort of U.S. women. Am J Epidemiol 1991;133:38-49.

31. Whelan EA, Sandler DP, Root JL, et al. Menstrual cycle patterns and risk of breast cancer. Am J Epidemiol 1994;140:1081—90.

Received for publication: 1 September 1997 